\title{
Microscale tipstreaming in a microfluidic flow focusing device
}

\author{
Shelley L. Anna a) and Hans C. Mayer \\ Department of Mechanical Engineering, Carnegie Mellon University, Pittsburgh, Pennsylvania 15213-3890
}

(Received 5 May 2006; accepted 3 August 2006; published online 8 December 2006)

\begin{abstract}
A microfluidic flow-focusing device is used to explore the use of surfactant-mediated tipstreaming to synthesize micrometer-scale and smaller droplets. By controlling the surfactant bulk concentration of a soluble nonionic surfactant in the neighborhood of the critical micelle concentration, along with the capillary number and the ratio of the internal and external flow rates, we observe several distinct modes of droplet breakup. For the most part, droplet breakup in microfluidic devices results in highly monodisperse droplets in the range of tens of micrometers in size. However, we observe a new mode of breakup called "thread formation" that resembles tipstreaming and yields tiny droplets in the range of a few micrometers in size or smaller. In this work, we characterize the growth of the thread and its maximum length as a function of flow variables and surfactant content, and we also characterize the period of droplet breakup as a function of these variables. Our results suggest possible methods for controlling the process. Using a simple flow visualization experiment as the basis, we report on preliminary efforts to model the thread formation process. (C) 2006 American Institute of Physics. [DOI: 10.1063/1.2397023]
\end{abstract}

\section{INTRODUCTION AND BACKGROUND ON TIPSTREAMING}

The ability to synthesize highly monodisperse droplets, bubbles, and particles of controlled size has numerous potential applications in areas such as production of precision emulsions, foams, and suspensions, drug delivery, catalysis, and medical imaging. Microfluidics appears to offer a promising new route to fabrication of these materials, enabling the production of highly uniform droplets, bubbles, and particles in the 10-100 micrometer size range. By flowing two or more immiscible liquids into a microfluidic device, numerous authors have demonstrated the ability to make emulsion droplets, ${ }^{1-3}$ gas bubbles, ${ }^{4,5}$ solid polymeric particles, ${ }_{9}^{6-8}$ and even more novel particles including double emulsions, ${ }^{9}$ polymeric particles with arbitrary nonspherical shapes, ${ }^{10}$ spherical colloidal shells, ${ }^{11}$ and Janus particles. ${ }^{10}$ The highly ordered packing of the monodisperse droplets has also been used to template porous materials. ${ }^{12}$

Two principal geometries are used for droplet formation: (a) a T-shaped junction, ${ }^{2}$ at which viscous shear stresses from the continuous stream of one liquid overcome surface tension at the liquid-liquid interface and pull off droplets of a second immiscible stream, and (b) a flow-focusing geometry, ${ }^{1,4}$ in which strong elongational flow of a continuous stream of one liquid flowing through a constriction draws a thin filament of the second immiscible liquid through the orifice. This filament subsequently breaks into droplets. Despite the promise of these small-scale devices, the droplet and particle sizes that can be produced are rarely smaller than 5-10 micrometers in diameter, largely because the droplet breakup mechanism is strongly influenced by the smallest feature size in the device (i.e., the constriction

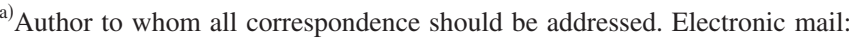
sanna@cmu.edu
}

orifice or junction geometry). ${ }^{13}$ At the present time, microfluidic techniques have not been widely extended for synthesis of droplets that are a few micrometers in size or smaller. Nonmicrofluidic methods are available for synthesis at this length scale, for example electrohydrodynamic jetting has been used to produce submicrometer scale fibers and particles $;{ }^{14}$ however, this method is limited in the choice of materials and in the range of operating conditions possible. This method has been implemented recently in a microfluidic device to produce particles ranging from 1 to 10 micrometers in size ${ }^{15}$ however, the resulting particles were highly polydisperse compared with other microfluidic drop formation methods described above.

The present paper reports a new microfluidic method for production of submicrometer and potentially nanoscale droplets and particles. In this method, the elongational flow in a flow-focusing microfluidic device is used along with dissolved surfactants in one of the liquid phases to create strong surface tension gradients. In contrast with past work using this geometry, in which liquids contained either no added surfactant, ${ }^{4}$ or a very large amount of surfactant, ${ }^{1,2,12,16}$ the present paper focuses on moderate concentrations of wellcharacterized surfactants. A key observation is that the surfactant concentration plays a central role in the mode of droplet formation. Under the right experimental conditions we observe that the droplet forms a conical shape with a highly sharpened tip, and that tiny liquid threads and droplets stream off the end of that tip. Our observations resemble a phenomenon called "tipstreaming" that has been reported previously in larger scale isolated droplets in unbounded linear flows. In the present paper, we investigate the microscale tipstreaming behavior in detail and explore possibilities to control the phenomenon as a possible route to micrometerscale and smaller droplets.

The tipstreaming phenomenon was first reported by Taylor. ${ }^{17}$ In these experiments, a large isolated droplet ap- 
proximately $1.6 \mathrm{~mm}$ in diameter is exposed to either a pure shear flow or a pure elongational flow in a four-roll mill. At particular values of the capillary number, the droplet becomes elongated and the ends appear to be pointed. Tiny droplets less than 50 micrometers in diameter (more than an order of magnitude smaller than the parent droplet) stream from the pointed ends. ${ }^{18}$ This behavior was observed to be transient, and subsequent experimental work speculated on a number of possible sources of this transient behavior, including the possibility that as the ejected droplets reduce the size of the parent droplet, the capillary number also decreases to a value outside the relevant region for tipstreaming. ${ }^{19}$ More recently, de Bruijn established that the phenomenon occurs as a result of impurities present on the liquid-liquid interface, and more importantly, that there is an optimal range of surfactant bulk concentrations for which tipstreaming can occur. ${ }^{20}$ Within this range, the tipstreaming drop size increases as the surfactant concentration increases, a finding consistent with more recent experiments of Janssen et al. ${ }^{21-23}$ and $\mathrm{Hu}$ et al., who specifically investigated the influence of copolymer at interfaces. ${ }^{24}$ De Bruijn confirmed that the tiny droplets are saturated with surfactant by showing that their surface tension values are very low compared with that of the parent droplet. Thus, the observation that tipstreaming is transient is likely due to the tiny droplets carrying away the surfactant, leaving the interface depleted of surfactant. A recent review article by Stone ${ }^{18}$ summarizes the necessary conditions for tipstreaming to occur. For example, the capillary number is always observed to have a value of approximately $\mathrm{Ca}=\mu_{o} G a / \gamma \approx 0.5$, where $\mu_{o}$ is the viscosity of the outer liquid, $G$ is the effective elongational or shear rate, $a$ is the radius of the undeformed parent droplet, and $\gamma$ is the interfacial tension. In addition, the viscosity ratio between the two liquids must be very low, $\lambda=\mu_{i} / \mu_{o} \leq O(0.1)$. Despite these advancements in our understanding of the tipstreaming phenomenon, most of the experimental work to date has not used well-characterized surfactants, making it difficult to further investigate mechanisms for tipstreaming.

A basic physical model for surfactant-mediated tipstreaming is illustrated in Fig. 1. When surfactant molecules are dissolved in a liquid, those molecules can diffuse from the bulk toward an interface, and molecules in close proximity to the interface can also adsorb onto and desorb from the interface. The equilibrium surface concentration of molecules and the rate at which the equilibrium concentration is reached depend upon the mass transfer rates for each of these mechanisms. As the parent droplet is subject to a strong local strain rate (elongational or shear flow), surfactant molecules present on the surface are swept by convection toward the pole of the droplet. If the rate of compression of the surfactant molecules exceeds the rate at which molecules can desorb from the interface, surfactant can accumulate at the pole. Large surface concentrations at the pole allow the local surface tension to drop nearly to zero, enabling the surface curvature at the pole to attain a very high value, appearing pointed. Subsequently, the local strain field draws thin threads of the droplet liquid from the tip, and those threads then break into droplets comparable in size to the original thread by a Rayleigh capillary breakup mechanism. These

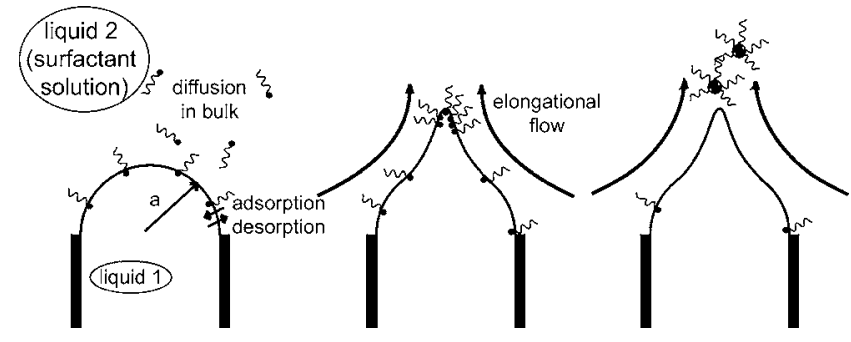

FIG. 1. Schematic illustration of the tipstreaming mechanism. Surfactants are dissolved in the outer liquid and molecules can diffuse to the interface and adsorb onto or desorb from the interface. Elongational flow past the droplet situated at the end of a capillary tube stretches the interface and leads to gradients of surfactant concentration along the interface. Under conditions in which surfactants cannot desorb fast enough compared with their rate of compression along the interface due to the elongational flow, the tip of the droplet can become packed with surfactants. This leads to a sharp decrease in the interfacial tension and thus a highly curved tip. Eventually the highly curved tip is drawn into a thin thread, which subsequently breaks into tiny surfactant-laden droplets. The interface is temporarily depleted of surfactant molecules. We note that our experiments utilize surfactant in the inner fluid; however, we do not expect this difference to change the basic physical mechanism.

tiny droplets contain very large surfactant concentrations, and the parent droplet interface is temporarily depleted of surfactant molecules.

The above arguments were proposed in part by de Bruijn, ${ }^{20}$ and subsequent numerical simulations performed by Eggleton et al..$^{25}$ confirmed that the basic sequence of events does occur with insoluble surfactants if the appropriate surfactant mass transport equations are solved simultaneously with the Stokes equations in each liquid. These numerics are based upon a boundary integral formulation that was extended to include surfactant dynamics by Stone and Leal $^{26}$ and Milliken et al. ${ }^{27}$ While Eggleton and co-workers focused on elongational flow, recent work by Bazhlekov et $a l .^{28}$ using similar numerical methods has also shown similar behavior in droplets subject to shearing flows, also using insoluble surfactants. It is worth noting that this recent work reproduces numerically an experimental result reported by Grace $^{29}$ showing that below a critical viscosity ratio, surfactants lead to a sharp reduction in the critical capillary number for breakup of sheared droplets, and that this critical capillary number is independent of viscosity ratio below the critical value. Other theoretical work investigating tipstreaming has focused on using slender body theory to predict the pointed shapes observed in tipstreaming. ${ }^{30}$ Numerical and theoretical work on tipstreaming to date has primarily utilized insoluble surfactants. The important variables for the process established through this work, in addition to the capillary number and viscosity ratio, are the initial dimensionless surface coverage $X$ and an elasticity number $E l$, reflecting the sensitivity of surface tension to the local surface concentration. By fitting de Bruijn's measured surface tension values to a Langmuir model, ${ }^{31}$ we compute the corresponding range of dimensionless surface concentrations to be $0.1 \leq X \leq 0.9$ for the particular surfactant system used in that work. Importantly, the computed range of $X$ values coincides with that predicted by the numerical work of Eggleton et $a l^{25}$ To our knowledge, neither numerical simulations 


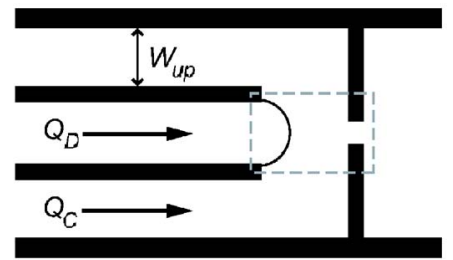

(a)

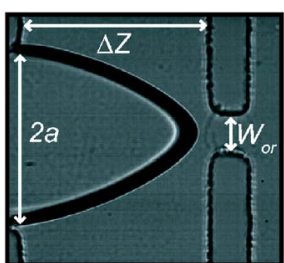

(b)
FIG. 2. Microfluidic flow focusing geometry. (a) Schematic diagram of the design denoting flow of both the inner and outer liquids from left to right. (b) Image of the orifice region of an actual microchannel [outlined with the dashed line in (a)], including a typical image of the water-oil interface extending toward the orifice from the upstream channel during a flow experiment. Dimensions shown are $W_{\text {up }}=280 \mu \mathrm{m}, a=90 \mu \mathrm{m}, \Delta Z=180 \mu \mathrm{m}$, and $W_{\mathrm{or}}=34 \mu \mathrm{m}$.

nor experiments investigating tipstreaming with wellcharacterized soluble surfactants have been reported.

In this paper, we present experimental observations of droplet formation in a microfluidic flow focusing device, focusing in particular on a new regime that we call "thread formation" in which thin threads substantially smaller than the minimum device feature size can be generated. Our experiments have yielded threads and droplets a few micrometers in diameter or less via a process resembling tipstreaming. In Sec. II, we describe the experiment and the particular surfactant solutions used to perform the present studies. Section III describes our observations, first describing observed droplet breakup modes as a function of dimensionless flow variables, then describing the key features of the new "thread formation" regime. We present quantitative measurements of the droplet breakup period in each of the major regimes. Finally, we discuss preliminary efforts to model aspects of the thread formation process.

\section{THE FLOW FOCUSING EXPERIMENT}

The experiments presented here utilize a flow focusing microfluidic device, shown in Fig. 2, that is similar to devices reported previously. ${ }^{1,4,32}$ Two immiscible liquids enter the device via three parallel microchannels, with the "continuous phase" liquid flowing in the outside two channels and the "dispersed phase" liquid flowing in the inner channel. Downstream, the inner microchannel ends and the three liquid streams come into contact just before entering a small orifice. Flow through the contraction leads to an elongationdominated strain field in the outer liquid, which then draws the inner liquid through the constriction, stretching the interface and leading to the formation of a liquid jet. The jet breaks into droplets.

We fabricate the device shown in Fig. 2 using soft lithography methods. ${ }^{33,34}$ The devices are molded in poly(dimethylsiloxane) (PDMS; Dow Sylgard 184). To close the device, the molded PDMS channel is covalently bonded to a glass microscope slide via exposure to RF plasma. Prior to bonding, the microscope slide is first coated with a thin film of PDMS in order to ensure that all microchannel walls have the same wetting characteristics. Exposure to the plasma renders the PDMS surfaces temporarily hydrophilic; we postbake the assembled microchannels at $150^{\circ} \mathrm{C}$ for several hours to ensure that the surface reverts to a hydrophobic condition. ${ }^{35}$ Due to the hydrophobic wetting condition, we use oil as the continuous outer liquid and water as the dispersed inner liquid. The oil used in all of the experiments reported here is light mineral oil (Fisher Scientific) with a viscosity of $\mu_{o}=40 \mathrm{mPa}$ s as measured in a Brookfield DV-II+ Viscometer. The measured viscosity is consistent with the range specified by the manufacturer. The aqueous inner phase contains dissolved surfactants with varying bulk concentrations; we describe these solutions and their characteristics later in this section. All of the surfactant concentrations are low enough that the viscosity of the aqueous phase is not affected. Thus the viscosity ratio for all experiments reported here is fixed at a value of $\lambda=\mu_{i} / \mu_{o}=1 / 40=0.025$, well below the limiting value for tipstreaming in experiments using isolated droplets. ${ }^{18}$

Flow in the microchannel device is driven using two syringe pumps (Harvard Apparatus PHD 2000 pumps), one pump driving each of the two immiscible liquids. After adjusting the flow rates, droplet breakup is allowed to come to equilibrium for several minutes. The contraction flow resulting from the presence of the downstream orifice results in a highly elongational flow in the neighborhood of the orifice. We can define a capillary number "Ca" based on the outer liquid viscosity and the effective elongation rate $G=\Delta V / \Delta Z$, where $\Delta V=V_{\text {constr }}-V_{\text {up }}$ is the difference between the smaller upstream velocity and the larger velocity inside the orifice, and $\Delta Z=180 \mu \mathrm{m}$ is the distance from the end of the inner microchannel to the entrance to the orifice. The capillary number is given by

$$
\mathrm{Ca}=\frac{\mu_{o} G a}{\gamma_{\mathrm{EQ}}}=\frac{\mu_{o} a \Delta V}{\gamma_{\mathrm{EQ}} \Delta Z}=\frac{\mu_{o} a Q_{C}}{\gamma_{\mathrm{EQ}} h \Delta Z}\left[\frac{1}{W_{\mathrm{OR}}}-\frac{1}{2 W_{\mathrm{UP}}}\right],
$$

where $Q_{C}$ is the outer liquid flow rate and $\gamma_{\mathrm{EQ}}$ is the equilibrium surface tension corresponding to the surfactant concentration utilized. We describe our method for measuring values of $\gamma_{\mathrm{EQ}}$ in the following paragraphs. Microchannel dimensions, $W_{\mathrm{OR}}=34 \mu \mathrm{m}$ and $W_{\mathrm{UP}}=280 \mu \mathrm{m}$, are labeled in the figure. We note that the microchannel design is nominally planar, so that the depth of the entire device has a uniform value of $h=98 \mu \mathrm{m}$. The dimension $a$ denotes the half-width of the upstream inner microchannel, which is analogous to the parent drop radius in experiments involving isolated droplets.

The aqueous surfactant solutions used in the present experiments are prepared using $\mathrm{C}_{12} \mathrm{E}_{8}$ (Sigma Aldrich P8925$1 \mathrm{G}$, used as received), a nonionic polyethoxylated surfactant that is soluble in water. The chemical structure of this molecule is $\left(\mathrm{CH}_{3}\left(\mathrm{CH}_{2}\right)_{\mathrm{i}}\left[\mathrm{O}\left(\mathrm{CH}_{2}\right)_{2}\right]_{\mathrm{j}} \mathrm{OH}\right)$, where $i=12$ and $j=8$ are the lengths of the carbon tail group and the ethoxylated head group, respectively. Solutions are prepared using deionized water obtained from a Millipore purification system (18 M $\Omega$, RO feed, 0.2 micrometer filtration). Quantities of surfactant are measured by an Ohaus Adventurer electronic balance and mixed using volumetric flasks containing deionized water in order to produce known concentrations of aqueous surfactant solution. Properties of the $\mathrm{C}_{\mathrm{i}} E_{\mathrm{j}}$ surfactant family have been thoroughly characterized in the literature at 


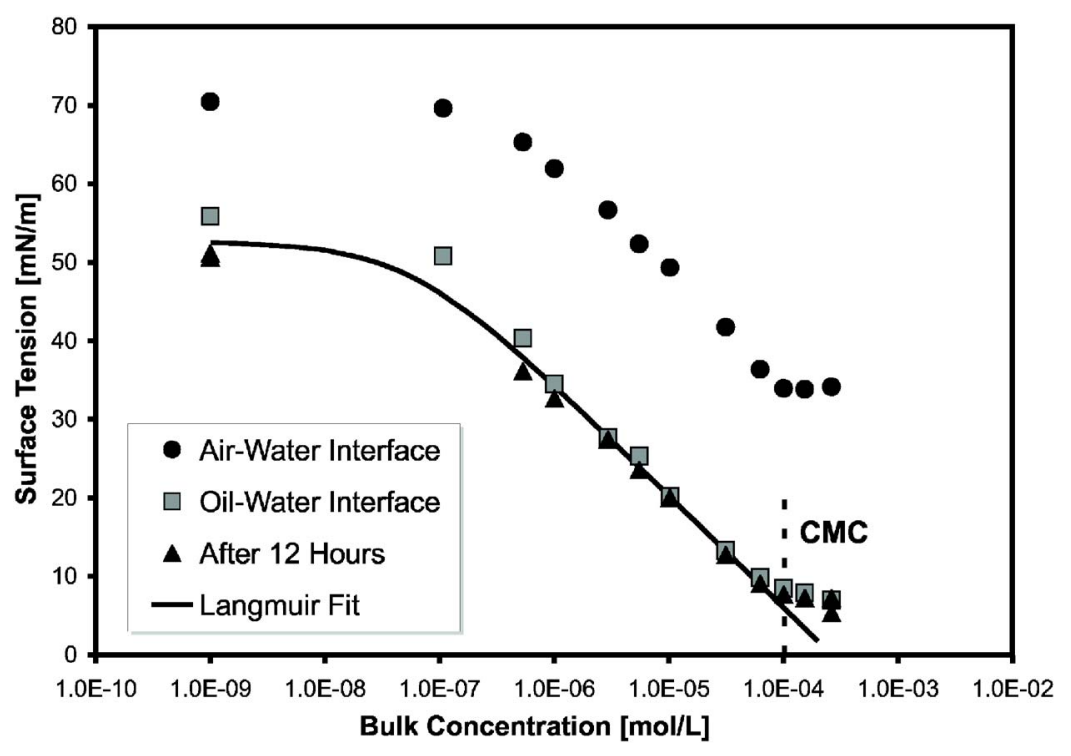

FIG. 3. Surface tension measurements at air-surfactant solution and oil-surfactant solution interfaces as a function of bulk concentration of $\mathrm{C}_{12} \mathrm{E}_{8}$ surfactant. Measurements were performed using a DuNuoy ring tensiometer and were compared with measurements after 12 $\mathrm{h}$ to ensure that equilibrium had been reached. The solid curve represents a fit of the measured data to the Langmuir model. The critical micelle concentration (CMC) is deduced from the measured surface tension data and is denoted on the graph.

air-water interfaces, ${ }^{36}$ including detailed pendant bubble measurements of the dynamic surface tension from which mass transfer coefficients such as the diffusion coefficient and the adsorption and desorption rate constants have been extracted. ${ }^{37-39}$ Thus these surfactants are good candidates for study of surfactant-mediated tipstreaming in microfluidic devices, enabling a systematic investigation of the role of different mass transfer mechanisms in the process. A wide variation in surfactant solution properties such as critical micelle concentration, equilibrium surface tension, and equilibrium constants, which contain adsorption and desorption coefficients, can be achieved by adjusting the lengths of the ethoxylated head groups and carbon tail groups while maintaining the same general chemistry of the surfactant molecule. $^{36,40}$

Although surface tension and mass transfer coefficients have been characterized thoroughly for air-water interfaces, there is little information available for oil-water systems. To obtain some information about the properties of the mineral oil-water interface, we measure the equilibrium surface tension $\gamma_{\mathrm{EQ}}$ as a function of surfactant bulk concentration using a DuNuoy ring tensiometer (Cenco). The surface tension curves are shown in Fig. 3. We measure the surface tension at both the air-water and oil-water interfaces for bulk concentrations ranging from $C_{\infty}=0$ (no surfactant) to a value somewhat greater than the critical micelle concentration. From these measurements, we obtain the value of the critical micelle concentration, $\mathrm{CMC}=1.0 \times 10^{-4} \mathrm{~mol} / \mathrm{L}$, as the value at which the surface tension reaches a constant value. Surface tension values measured for the air-water interface agree well with values reported in the literature. ${ }^{41}$ Values of the CMC obtained from both air-water and oil-water surface tension measurements are in good agreement, as expected. To ensure that the surface tension has reached an equilibrium value, we also measure the interfacial tension after $12 \mathrm{~h}$ and we observe very little change. A measurement at long times is also useful to determine whether any partitioning of the surfactant into the oil phase over time may influence the surface properties. We infer from the measurements shown in
Fig. 3 that such partitioning is of negligible importance, especially at the much shorter, microsecond time scales relevant to our experiments. We fit the measured equilibrium surface tension to a Langmuir model, given by

$$
\gamma_{\mathrm{EQ}}=\gamma_{O}+R T \Gamma_{\infty} \ln (1-X)
$$

where $\gamma_{0}$ is the clean interface value, $R$ is the ideal gas constant, and $T$ is the temperature. $X$ is the dimensionless surface concentration, defined by

$$
X=\frac{\Gamma_{\mathrm{EQ}}}{\Gamma_{\infty}}=\frac{C_{\infty}}{C_{\infty}+a},
$$

where $\Gamma_{\mathrm{EQ}}$ is the equilibrium surface concentration and $\Gamma_{\infty}$ is the maximum packing fraction of the surfactant molecules at the surface. This ratio can also be defined in terms of the bulk concentration $C_{\infty}$ and the parameter $a$, which is an equilibrium coefficient also related to the adsorption and desorption rate coefficients. The dimensionless surface concentration is one of the essential parameters for tipstreaming identified through recent numerical simulations. ${ }^{25,28} \mathrm{We}$ obtain fitted values of $\Gamma_{\infty}=2.5 \times 10^{-6} \mathrm{~m}^{2} / \mathrm{mol}$ and $a=5.4$ $\times 10^{-8} \mathrm{~mol} / \mathrm{L}$, and we find that the surface tension decreases from a clean interface value of $\gamma_{0}=52.5 \mathrm{mN} / \mathrm{m}$ to a minimum value at the CMC of $\gamma_{\min }=7.5 \mathrm{mN} / \mathrm{m}$. In calculating dimensionless variables corresponding to our experiments, we use the equilibrium surface tension $\gamma_{\mathrm{EQ}}$ corresponding to the bulk concentration $C_{\infty}$ of a given surfactant solution.

In the flow experiments reported here, we control the flow rate of each liquid and the bulk surfactant concentration. Thus, in dimensionless terms, we explore a threedimensional phase space consisting of (i) the capillary number $\mathrm{Ca}$, (ii) the flow rate ratio $\varphi$, given by

$$
\varphi=Q_{C} / Q_{D}
$$

where $Q_{D}$ is the volume flow rate of the inner liquid, and (iii) the ratio of bulk surfactant concentration to the critical micelle concentration, $C_{\infty} / \mathrm{CMC}$. We vary the surfactant bulk concentration in our experiments from $0 \leq C_{\infty} / \mathrm{CMC} \leq 14.5$. 
TABLE I. $\mathrm{C}_{12} \mathrm{E}_{8}$ surfactant solutions used in flow focusing experiments. Bulk concentration (dimensional) is reported along with the dimensionless bulk concentration normalized by the critical micelle concentration, and the surface coverage, $X$, computed from fits of surface tension to the Langmuir isotherm. Values of equilibrium surface tension at each concentration are also listed.

\begin{tabular}{lcccc}
\hline \hline Solution No. & Weight $\%$ & $X$ & $C_{\infty} / C_{\mathrm{CMC}}$ & $\gamma_{\mathrm{EQ}}(\mathrm{mN} / \mathrm{m})$ \\
\hline 1 & 0 & 0 & 0 & 52.5 \\
2 & $8.6 \times 10^{-6}$ & 0.746 & 0.0016 & 44.0 \\
3 & $2.7 \times 10^{-3}$ & 0.9988 & 0.5 & 10.8 \\
4 & $1.4 \times 10^{-2}$ & 0.9999 & 2.6 & 7.5 \\
5 & $4.3 \times 10^{-2}$ & 0.9999 & 8.0 & 7.5 \\
6 & $7.8 \times 10^{-2}$ & 0.9999 & 14.5 & 7.5 \\
\hline \hline
\end{tabular}

Corresponding values of weight fraction, dimensionless surface concentration, and equilibrium surface tension are given in Table I for the six different surfactant solutions that we consider here. We note that the value of dimensionless surface concentration $X$ rapidly approaches unity as the bulk concentration approaches the CMC. Thus for most of our experiments, especially those exhibiting the most interesting droplet breakup behavior, the value of $X$ is not a good indicator of changes in the amount of surfactant present. Thus we choose to report the value of bulk concentration instead in order to accurately reflect the experimental conditions. We do not explicitly consider the influence of micelles on the dynamics of the process we describe here, although there is evidence that $\mathrm{C}_{12} \mathrm{E}_{8}$ forms small spherical micelles over a wide range of concentrations above the $\mathrm{CMC}^{42}$ At larger concentrations, the dynamics of breaking and reforming micelles near the interface may introduce additional time scales into the transport of surfactants to the oil-water interface.

As the flow rate ratio $\varphi$ increases, the outer liquid flows significantly faster than the inner liquid, thus mimicking the case of an isolated droplet in which the droplet phase liquid is stationary. In our experiments, the outer liquid flow rate ranges from $0.335 \leq Q_{C} \leq 41.9 \mathrm{~mL} / \mathrm{h}$. To estimate the relative importance of inertia in our experiments, we define a Reynolds number, given by

$$
\operatorname{Re}_{C}=\frac{\rho_{o} V_{\text {up }} W_{\text {up }}}{\mu_{o}}=\frac{\rho_{o} Q_{C}}{h \mu_{o}},
$$

where $\rho_{o}$ is the density of the outer liquid. Defined in this way, the Reynolds number in the outer liquid ranges from $10^{-2} \leq \operatorname{Re}_{C} \leq 3$. We vary the flow rate ratio from $40 \leq \varphi$ $\leq 320$, which means that the Reynolds number in the inner liquid, defined by

$$
\operatorname{Re}_{D}=\frac{\rho_{i} Q_{D}}{h \mu_{i}}=\frac{\left(\rho_{i} / \rho_{o}\right)}{\lambda \varphi} \operatorname{Re}_{C},
$$

ranges from $10^{-3} \leq \operatorname{Re}_{D} \leq 3$, where $\rho_{i}$ is the density of the inner liquid. In addition, we define a Weber number, defined by the ratio of inertia to capillary forces,
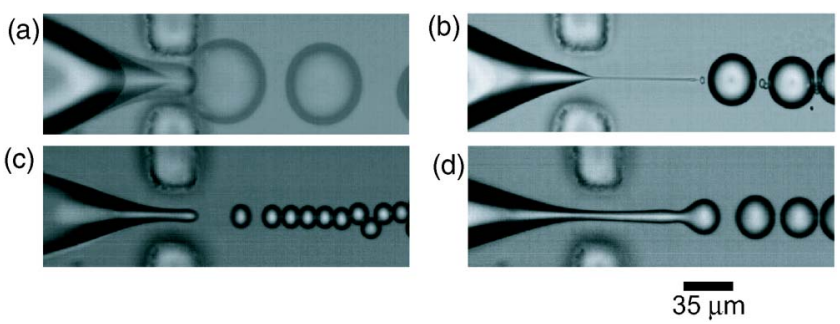

FIG. 4. Representative images of the four main droplet formation modes observed in the flow focusing microfluidic device, in order of appearance as capillary number increases. Note that panel (b) corresponds to a higher capillary number since the relevant range of capillary numbers for each mode increases with bulk surfactant concentration. (a) "Geometrycontrolled" breakup $(\mathrm{Ca}=0.05, \varphi=160, X=0)$. In this case, a series of images, consecutive in time, have been overlaid to show that the tip of the main water droplet periodically extends and retracts during the process. The other modes are (b) "thread formation" $(\mathrm{Ca}=0.5, \varphi=40, X \approx 1)$, (c) "dripping" $(\mathrm{Ca}=0.15, \varphi=80, X=0)$, and (d) "jetting" $(\mathrm{Ca}=0.3, \varphi=40, X=0)$.

$$
\mathrm{We}_{C}=\frac{\rho_{o} V_{\mathrm{up}}^{2} W_{\mathrm{up}}}{\gamma_{\mathrm{EQ}}}=\frac{\rho_{o} Q_{C}^{2}}{2 W_{\mathrm{up}} h^{2} \gamma_{\mathrm{EQ}}} .
$$

The maximum value of the Weber number in our experiments is $\mathrm{We}_{C} \approx 3 \times 10^{-4}$. Thus, we assume that inertia can be neglected in our experiments, although we recognize that inertia will play an increasingly important role at the highest flow rates.

During flow experiments, we image the liquid-liquid interface using a high-speed monochrome digital camera (IDT XS-4) that is interfaced with an inverted light microscope (Nikon TE2000U). Drop sizes, thread lengths, and drop breakup frequencies are measured using the image processing toolbox in Matlab. Frequency of droplet formation is obtained via manual frame counting, where the frame rate for the acquisition is more than twice the frequency of drop breakup to avoid aliasing effects.

\section{RESULTS AND DISCUSSION}

\section{A. Phase diagrams and modes of droplet breakup}

For each of the six surfactant solutions listed in Table I, we perform flow focusing experiments as described above, varying the flow rate of both the inner and outer liquids. We observe droplet breakup characteristics as a function of capillary number $\mathrm{Ca}$, defined as in Eq. (1), and flow rate ratio $\varphi$, defined as in Eq. (4). We categorize our observations into four distinct droplet breakup regimes, for which representative images are shown in Fig. 4. Three of those droplet breakup modes are observed at all surfactant concentrations including the clean interface case, namely "geometrycontrolled" breakup [Fig. 4(a)], "dripping" [Fig. 4(c)], and "jetting" [Fig. 4(d)]. In a specific range of surfactant concentrations we also observe a fourth mode of breakup that we call "thread formation," shown in Fig. 4(b). We describe this fourth mode in further detail in Sec. III A.

The geometry-controlled regime is characterized by primary droplet sizes that are roughly equal to the orifice size. The droplet size is highly monodisperse (polydispersity $<2 \%$ ) and nearly constant as a function of both the capillary number and the flow rate ratio. Droplets break off from the 
dispersed phase "finger" via the following mechanism, which has been investigated in detail by Garstecki. ${ }^{13}$ Initially, the dispersed phase liquid is drawn into the orifice, forcing the continuous phase liquid to flow in a narrow region around the interface. To maintain the applied flow rate, a higher pressure is needed in the continuous liquid stream in order to drive flow through this narrow region. The higher upstream pressure leads to pinching of the interface, which progresses until a discrete droplet separates from the initial finger. Once the droplet has formed, the finger of dispersed phase liquid retracts to a position upstream of the orifice. This sequence of events is shown in Fig. 4(a), in which several sequential images have been superimposed to demonstrate the position of the interface as the droplet pinches off. Although the droplet size is nearly constant, the frequency of breakup changes in order to satisfy conservation of mass of the inner liquid stream. These trends will be described in more detail in the next section.

Increasing the capillary number leads to droplet formation in the dripping regime. Here, the dispersed phase finger does not retract after a droplet pinches off, but rather remains at a fixed location inside the orifice during the entire process. In this case, the droplets are somewhat smaller than the orifice size, and the dispersed phase finger is longer and narrower than that observed in geometry-controlled breakup, as shown in Fig. 4(c). The droplet size distribution again appears highly monodisperse and the droplet diameter decreases as both the capillary number and the flow rate ratio increase. The dripping mode of breakup is similar to that observed by Utada et al. ${ }^{9}$ in the formation of double emulsions, although the geometry in their case is axisymmetric and overall dimensions are larger than in our flow focusing device. Xu et al. also observe a similar mode of breakup. ${ }^{43}$ In this case, the dispersed phase finger will break into discrete droplets when the finger becomes sufficiently elongated to sustain a Rayleigh capillary instability. ${ }^{44}$ From the theoretical arguments of Tomotika for liquid threads in viscous liquids, ${ }^{45}$ we may expect that the resulting droplet radius will be approximately double the radius of the liquid thread, $R_{\text {drop }}=2.1 R_{\text {thread }}$, for a viscosity ratio of $\lambda=0.025$. Our measurements yield values ranging from $1.9 \leq R_{\text {drop }} / R_{\text {thread }}$ $\leq 2.5$, consistent with the prediction. The dripping mode of breakup thus yields droplets smaller than the minimum device feature size, although the minimum droplet size still appears to be limited to approximately one order of magnitude smaller than the orifice size.

The dripping mode gives way to jetting as the capillary number continues to increase. In this case, the dispersed phase finger extends beyond the exit of the orifice and resembles a long viscous jet, as shown in Fig. 4(d). Again, the jet breaks into discrete droplets due to a Rayleigh capillary instability, but in this case the droplet size is larger than in dripping mode, and can be as large as the orifice width or larger. In the jetting regime, droplet size distributions are not monodisperse and the droplet breakup appears to be less controlled.

When no surfactants are present in the aqueous phase $\left(C_{\infty} / \mathrm{CMC}=0 ; X=0\right)$, we observe a progression in the droplet breakup modes described above as the capillary number in- creases. Geometry-controlled breakup occurs at the lowest capillary numbers. Around a capillary number of $\mathrm{Ca} \approx 0.1$, we observe a transition to dripping. Further increasing the capillary number, we observe a transition to jetting at a capillary number around $\mathrm{Ca} \approx 0.2$. The dependence of the transitions on flow rate ratio is much weaker. As the flow rate ratio increases, the critical capillary numbers at which transitions occur shift to slightly higher values. For example, at a flow rate ratio of $\varphi=40$, the transition from geometrycontrolled breakup to dripping occurs at a value of $\mathrm{Ca}=0.09$, while the transition occurs at a slightly larger value of $C a=0.15$ when the flow rate ratio is $\varphi=1280$. These trends are shown in the phase diagram of Fig. 5(a).

As surfactant is added, the capillary numbers at which transitions occur increase. These critical capillary numbers increase as the surfactant concentration increases. At a surfactant concentration greater than the critical micelle concentration, $C_{\infty} / \mathrm{CMC}=2.56(X=0.9999)$, the maximum capillary number at which geometry-controlled breakup occurs ranges from $0.3 \leq \mathrm{Ca} \leq 0.4$ as the flow rate ratio increases. The transition from dripping to jetting occurs at a capillary number ranging from $1.2 \leq \mathrm{Ca} \leq 1.7$ as the flow rate ratio increases. We note that our definition of the capillary number already utilizes the equilibrium surface tension $\gamma_{\mathrm{EQ}}$ [cf. Eq. (1)], which accounts for the decrease in surface tension due to added surfactant. Thus, we believe that this shift to larger critical capillary numbers reflects the importance of nonequilibrium surfactant effects on droplet breakup mechanisms. ${ }^{46}$

In addition to the three regimes described thus far, a fourth mode of droplet breakup is also observed. We call this mode of breakup "thread formation," and we observe this mode at capillary numbers intermediate between geometrycontrolled breakup and dripping. As shown in Fig. 4(b), large droplets comparable in size to those observed in geometrycontrolled breakup still form, but in between these larger droplets, thin liquid threads are drawn from the tip of the dispersed phase finger, which appears to form a sharp point during the formation of the thread. The threads subsequently break into tiny droplets, presumably via a Rayleigh capillary instability. Thread formation occurs within a specific range of capillary numbers, $0.4 \leq \mathrm{Ca} \leq 1.0$, for a surfactant concentration of $C_{\infty} / \mathrm{CMC}=2.56$ as shown in the phase diagram of Fig. 5(b). We note that thread formation only occurs above a limiting value of the bulk surfactant concentration of $C_{\infty} / \mathrm{CMC} \geq 0.5$. We note that the range of capillary numbers in which thread formation is observed coincides with that at which tipstreaming occurs in isolated droplets subject to elongational and shear flows. ${ }^{18}$

\section{B. Thread formation}

When surfactants are present within a specific concentration range, and in a range of capillary numbers intermediate between geometry-controlled breakup and dripping, we observe a new mode of droplet breakup that to our knowledge has not been reported previously. In some ways, this regime resembles the geometry-controlled breakup regime, in that primary droplets are formed that are comparable in size to the width of the flow-focusing orifice. On the other 

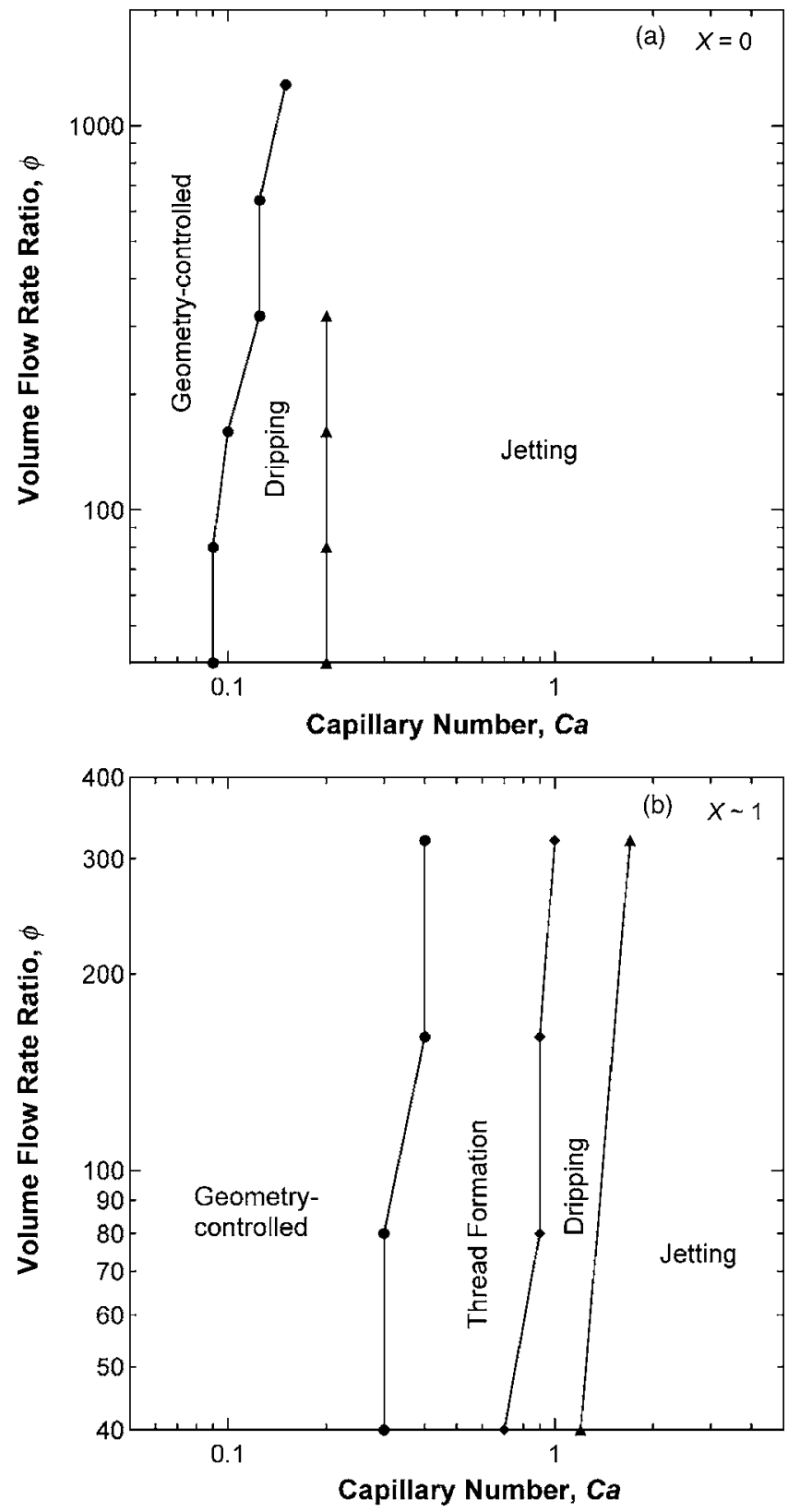

FIG. 5. Representative phase diagrams depicting regimes of capillary number and flow rate ratio in which different droplet formation modes are observed. (a) Observed droplet breakup regimes corresponding to the clean interface case, $X=0$. (b) Observed droplet breakup regimes corresponding to a bulk concentration of $C_{\infty}=2.56 \mathrm{CMC}$, which results in a dimensionless surface coverage of $X=0.9999$.

hand, we also observe the formation of very thin threads, which are drawn out from the pointed tip of the inner liquid thread subsequent to the breakup of each larger drop. Thus, at least at lower flow rate ratios, the formation of a thread is always preceded by the formation of a large droplet. Figure 6 shows a time sequence of images depicting the formation and growth of these threads. The images were recorded using a high-speed camera, and the time interval between images is $\Delta t=180 \mu \mathrm{s}$. The images show that as soon as the large drop has pinched off, the inner liquid finger begins to elongate again. The new thread lengthens and thins, and eventually pinches into discrete droplets. The droplets resulting from

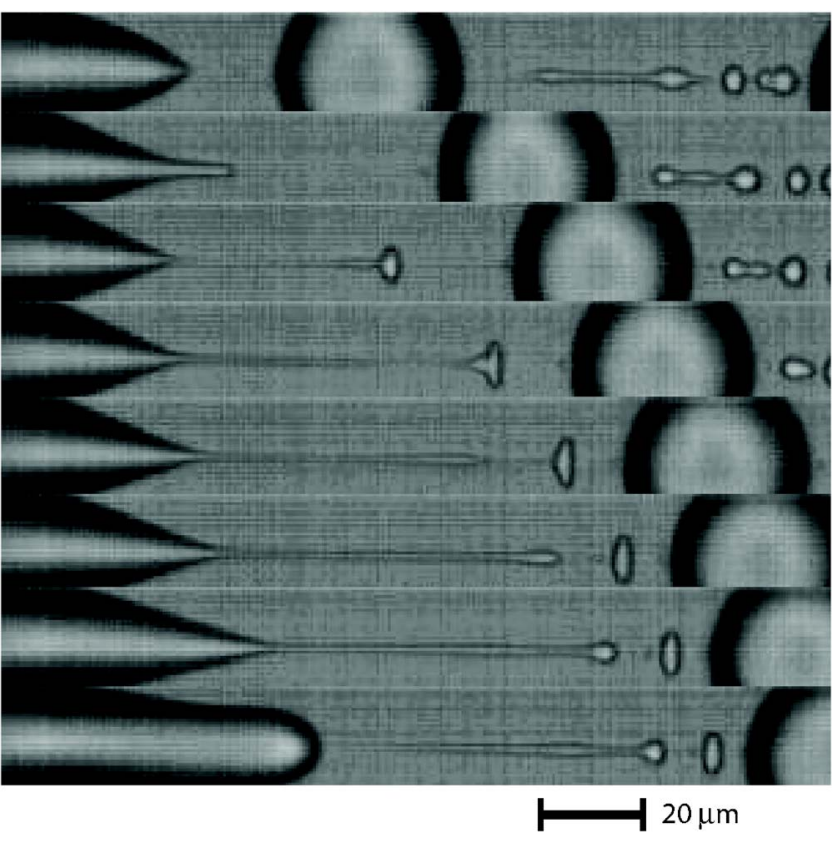

FIG. 6. A sequence of images in time showing the progression of thread formation. Images correspond to $\varphi=40 / 1, \mathrm{Ca}=0.5$, and $C_{\infty}=2.56 \mathrm{CMC}$. The time interval between images is $\Delta t=180 \mu \mathrm{s}$.

thread pinchoff are not always monodisperse, but are always significantly smaller than the large, primary droplet. The thin thread grows to a maximum length, after which the end of the inner liquid thread becomes rounded and another large droplet begins to pinch off. The process continues in a periodic manner.

As the flow rate ratio increases, the maximum extent of the intact thread increases and the thread diameter appears to decrease. The droplet sizes resulting from breakup of the thin threads decrease accordingly. Figures 7(a)-7(c) show a series of images of threads that have reached their maximum extent for flow rate ratios of $\varphi=40,80$, and 160 , illustrating the trend. In general, we observe that the maximum thread length increases as the inner liquid flow rate decreases, and also as the bulk surfactant concentration increases. The thread length peaks at a value of $\mathrm{Ca} \approx 0.6$. As the flow rate ratio increases still further, we observe a transition from thread formation with periodic formation of large droplets to a mode that more closely resembles tipstreaming in isolated droplets. As shown in Fig. 7(d) for a flow rate ratio of

\section{(a)}

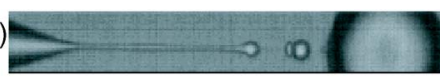

(b)

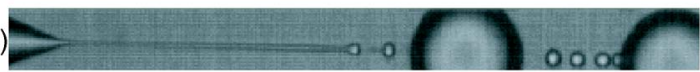

(c)

(d)

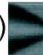

FIG. 7. Representative images of thread formation, depicting the maximum extent of the thread prior to the onset of significant capillary breakup. The thread length increases as the flow rate ratio $\varphi$ increases: (a) $\varphi=40 / 1$, (b) $\varphi=80 / 1$, (c) $\varphi=160 / 1$, (d) $\varphi=320 / 1$. 


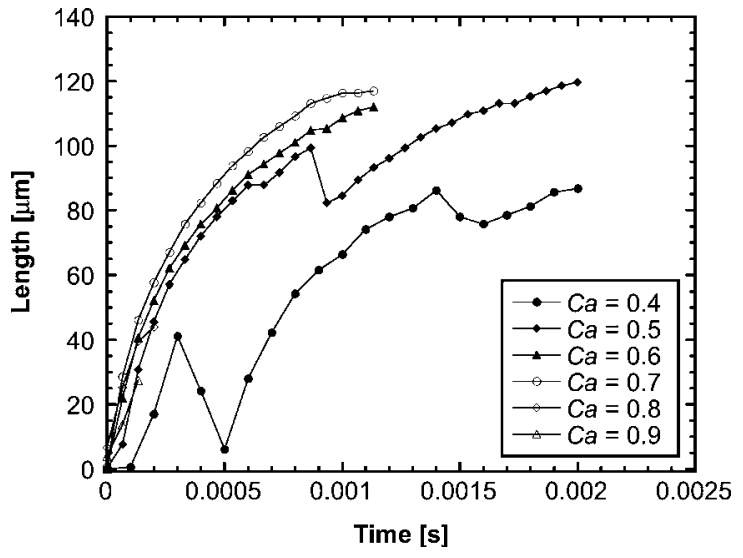

FIG. 8. Growth of the thread length with time for a fixed flow rate ratio, $\varphi=80 / 1$. Each curve represents a different capillary number ranging from $0.4 \leq \mathrm{Ca} \leq 0.9$. The initial time $t=0$ corresponds to the movie frame in which the thread first begins to extend. Thread length is shown up until the maximum thread length is reached, after which capillary breakup begins and the thread length decreases erratically.

$\varphi=320$, tiny droplets stream from the tip of the inner liquid thread for a relatively long period of time $\left(\Delta t_{\text {stream }}\right.$ $\approx 10-100 \mathrm{~ms}$ compared with $\Delta t_{\text {thread }} \approx 1-10 \mathrm{~ms}$ for threads at lower flow rate ratios). Streaming of the tiny droplets is preceded by the formation of several large droplets in succession. We observe "tipstreaming" when the flow rate ratio is large, in other words, when the inner liquid is flowing very slowly relative to the outer liquid. This limit is similar to the condition at which tipstreaming occurs in isolated, stationary droplets. We note that our current observations of droplet formation utilize optical microscopy, and thus the ability to resolve very small droplets is limited by the resolution of the microscope/objective/camera system (approximately 1-2 micrometers). As a result, the threads that form at higher flow rate ratios cannot be resolved in close proximity to the tip of the inner liquid thread, as Fig. 7 shows. Because of this, it is difficult to measure the thread length definitively and to resolve the details of the process by which the thread and the subsequent drops form. Nonetheless, the tiny droplets resulting from breakup of the thread are visible downstream, and these droplets appear to have diameters in the 1-2 micrometer range for $\varphi=320$. It is difficult to confirm whether these droplets have undergone coalescence in the upstream region where visualization is difficult.

The transient growth of the thin threads depends on the nature of the downstream flow field, and we expect it to be an important factor in the resulting size of the subsequent droplets. In the flow focusing geometry we consider here, the width of the microchannel downstream of the orifice is $W_{\text {down }}=834 \mu \mathrm{m}$. Thus the threads are extending into an expansion flow in which the velocity decreases from a large value inside the orifice to a smaller, but finite value corresponding to the total volume flow rate of liquid entering the expansion divided by the larger cross-sectional area of the downstream channel. Figure 8 shows measured values of the thread length as a function of time for several different capillary numbers, at a fixed flow rate ratio $(\varphi=80)$. The measurements show that the initial rate of growth of the thread increases as capillary number increases, and that growth slows at later times. This behavior is qualitatively consistent with the thread following the centerline of an expansion flow into a finite channel. The maximum thread length peaks at a value of $\mathrm{Ca}=0.5$ and decreases dramatically as the capillary number increases thereafter. In addition to its dependence on the downstream velocity field, the maximum extent of the intact thread also depends on the surfactant concentration. The presence of surfactants is expected to influence the stability of a liquid thread in a complicated manner. ${ }^{47}$

\section{Measurements of droplet formation frequency}

To further quantify the drop formation process in each regime described above, we measure the frequency of droplet formation as a function of capillary number, flow rate ratio, and surfactant bulk concentration. We expect the drop size and frequency to be related to one another through the volume flow rate of the inner liquid, at least for production of monodisperse droplets as in the geometry-controlled and dripping regimes. The relationship may be more complicated for a nonuniform size distribution such as that observed in the thread formation and tipstreaming regimes. For example, although it is evident that the large drops preceding thread formation use most of the volume of the input internal liquid, a large number of tiny droplets are also produced. Nonetheless, since the droplet sizes, especially those of the smallest droplets, are quite difficult to measure due to the limited resolution of optical microscopy, measurements of the frequency of formation can provide further insight into the behavior in each droplet formation regime.

Figures 9 and 10 show measurements of the time period $\tau_{\text {drop }}$ between the formation of primary droplets, as functions of capillary number, flow rate ratio, and surfactant concentration. These measurements correspond only to the largest primary droplets, and do not include satellite droplets or thin threads. Figure 9(a) shows the period as a function of the capillary number for several different surfactant concentrations, keeping flow rate ratio fixed. We observe that when little or no surfactant is present in the inner liquid stream, the time period between the formation of droplets decreases rapidly as the capillary number increases, falling by two orders of magnitude between $0.05 \leq \mathrm{Ca} \leq 0.2$. It is worth noting that this curve represents the transition between all three of the droplet breakup regimes observed when little or no surfactant is present. As the surfactant content increases, the period decreases significantly more slowly and the behavior of the period as a function of capillary number appears quite complicated, depending strongly on the given surfactant concentration. In particular, we note that in the capillary number range in which thread formation and tipstreaming occur, $0.4 \leq \mathrm{Ca} \leq 1.0$, the period for primary droplet formation increases, reaching a maximum at $\mathrm{Ca} \approx 0.5$. This is consistent with our observation that the maximum thread length also peaks at a similar value.

Figure 9(b) shows similar trends when the surfactant bulk concentration is kept fixed and the droplet formation period $\tau_{\text {drop }}$ is measured as a function of the capillary number for several different values of the flow rate ratio. The maxi- 

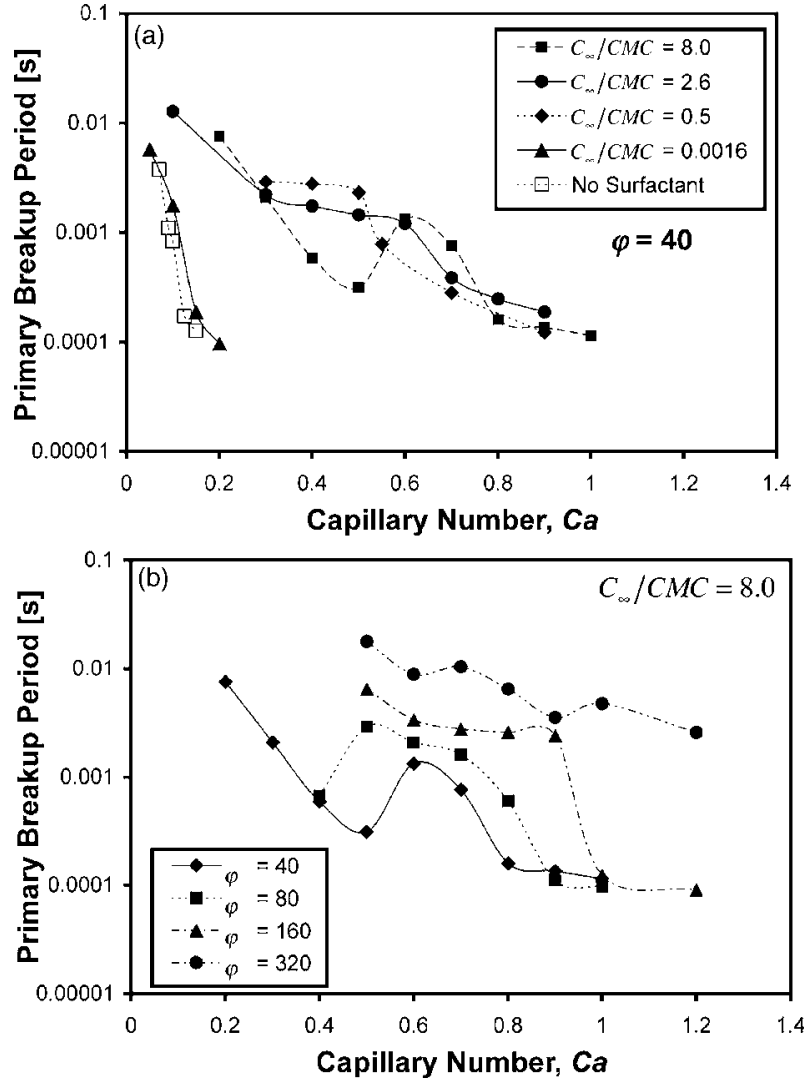

FIG. 9. Period of droplet formation (a) as a function of capillary number and concentration for a fixed flow rate ratio of $\varphi=40 / 1$, and (b) as a function of capillary number and flow rate ratio for a fixed value of surfactant concentration of $C_{\infty} / \mathrm{CMC}=8.0$. At a flow rate ratio of $\varphi=320 / 1$, sustained tipstreaming is observed for $0.5 \leq \mathrm{Ca} \leq 1.0$. The period is defined as the time interval between breakup of the primary drops, not accounting for threads or satellite droplets. This period is in general longer than, but comparable to, the thread formation period and the period of sustained tipstreaming.

mum period occurs at a value of approximately $\mathrm{Ca} \approx 0.5$ for all values of the flow rate ratio, but the maximum value itself increases as the flow rate ratio increases. In addition, the breadth of the peak also increases as the flow rate ratio increases, implying that long thin threads will occur over a wider range of capillary numbers for these flow rate ratios. Figure 10 summarizes the information presented in Figs. 9(a) and $9(\mathrm{~b})$, showing that the drop formation period $\tau_{\text {drop }}$ increases in general as flow rate ratio increases, even over a range of possible values of the capillary number. The corresponding droplet formation regimes are highlighted in the figure, emphasizing that the longest drop formation periods occur in the tipstreaming regime, and the shortest in the dripping regime. The period of droplet formation in the thread formation regime increases monotonically between these two extremes.

\section{A simplified model}

Thus far we have presented primarily experimental observations of a new droplet formation regime in microfluidic flow focusing devices in which thin threads form periodically, growing longer and thinner as the flow rate ratio increases. At large enough flow rate ratios, the thread forma-

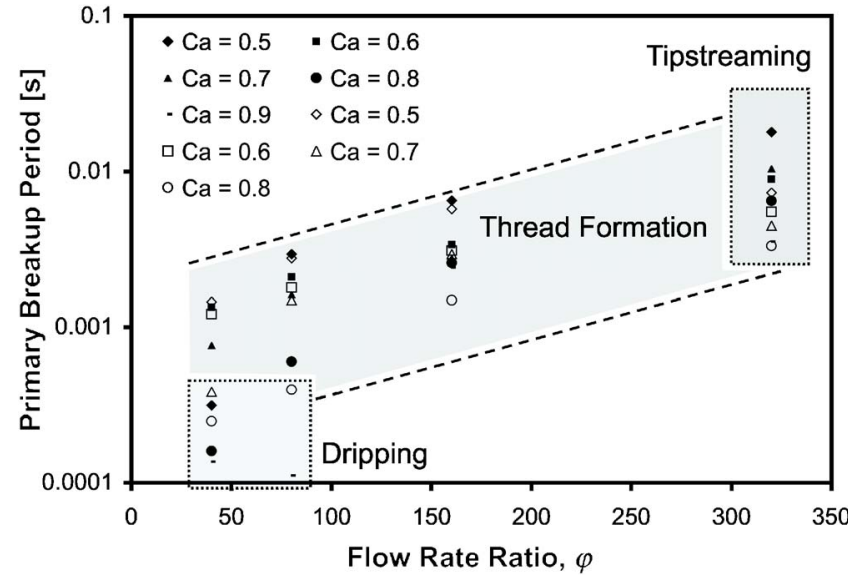

FIG. 10. Droplet formation period as a function of flow rate ratio and capillary number. Two different bulk concentrations are shown: open symbols represent $C_{\infty} / \mathrm{CMC}=2.6$ and closed symbols represent $C_{\infty} / \mathrm{CMC}=8.0$. Note that only at high flow rate ratios, e.g., $\varphi=320 / 1$, is tipstreaming observed. Thread formation is observed at flow rate ratios of less than $\varphi=320 / 1$. In certain instances, the droplet formation period in the thread formation regime appears to be bounded from below by that of the dripping regime.

tion regime gives way to tipstreaming of micrometer-scale droplets. This process offers a potential new route toward the production of micrometer- and submicrometer-scale droplets, but to gain control of this process, a detailed understanding of the mechanisms behind the tipstreaming process is needed. Modeling this process is not trivial since a detailed model for the problem involves simultaneous solutions of the Stokes equations in each liquid together with velocity and stress boundary conditions at the interface. ${ }^{25-28}$ The normal and shear stress boundary conditions couple the velocity field with variations in the surface tension along the interface, and additional equations are also needed in order to couple surface tension with surface concentration, and surface concentration with diffusion, adsorption, and desorption processes for mass transfer of molecules from the bulk onto the interface.

Solution of these equations is possible via detailed numerical simulations. For example, Jin et al. have modeled the gravity-driven pinchoff of bubbles in (soluble) surfactant solutions, demonstrating that the shape of the necked region and the eventual pinchoff of a bubble strongly depend on the surfactant concentration as well as the relative magnitudes of the rates of mass transfer compared with rates of surface divergence (compression and expansion) along the interface. ${ }^{46}$ In particular, the authors show that during pinchoff, surfactants are swept toward the necked region, at which the surface compression is greatest, leading to large surface concentrations in this region. The motion of the pinched droplet away from the necked region also results in an elongational flow along the centerline of the rear of the droplet, thus setting up potentially ripe conditions for tipstreaming-like behavior. In fact, this work suggests that a process akin to thread formation may occur at appropriate values of Péclet and Biot numbers, two dimensionless groups that describe the ratio of time scales for convection to diffusion, and the ratio of time scales for surface compression to 


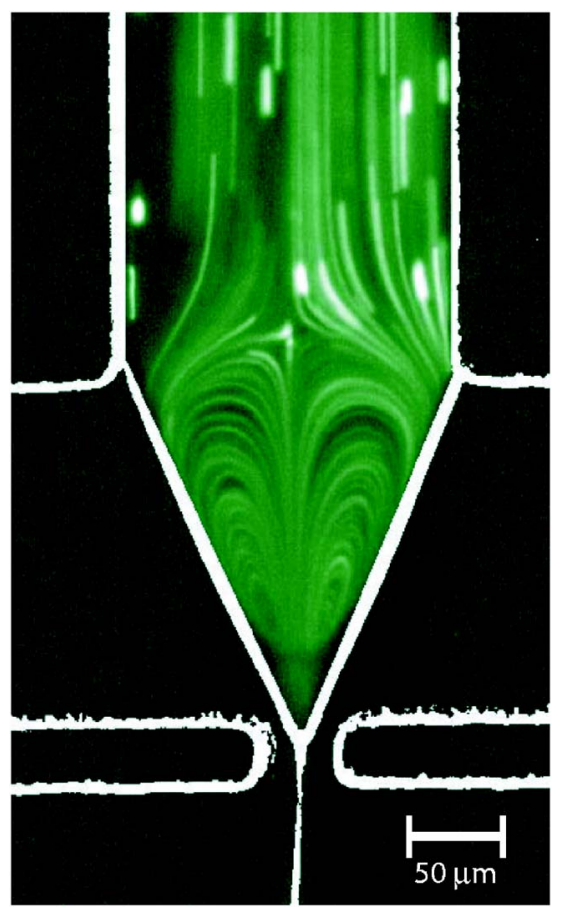

FIG. 11. Streamline image of fluorescent particles seeding the flow of the internal, aqueous liquid during thread formation $(\mathrm{Ca}=0.3, \varphi=100$, $C_{\infty} / \mathrm{CMC}=2.6$ ). The image shown is a time-lapsed image of the particle motions overlaid with a bright field image of the liquid-liquid interface, to which a threshold operation has been applied.

desorption of surfactant molecules, respectively. In other words, these parameters reflect the ability for diffusion and sorption processes to smooth out surface concentration gradients and counteract the steep surface concentration gradients needed for tipstreaming. It is worth noting that the flow field in the microfluidic flow focusing device, in which both inner and outer flow rates are controlled, imposes strong local rates of strain in the neighborhood of the necking region of the droplet. Thus it is expected that this flow will lead to significantly richer dynamics for droplet breakup in numerical simulations, just as we observe experimentally.

Our thread formation and tipstreaming observations also closely resemble recent work in which tipstreaming was observed as a result of a chemical reaction that produces surfactant at an oil-water interface. ${ }^{48}$ During pendant drop measurements of the dynamic surface tension, the authors observed that on occasions in which the pendant drop breaks off and settles in the outer liquid bath, this pinchoff is followed by tipstreaming of tiny droplets, in this case $\sim 4 \mathrm{mi}-$ crometers, for a sustained period of time. The authors offer physical arguments that this process results from strong surface tension gradients and they show that an apparent capillary number for the process is approximately $\mathrm{Ca} \approx 0.5$ as is expected for classic tipstreaming. In a follow-up paper, Krechetnikov and Homsy ${ }^{49}$ present analytical solutions for aspects of the tipstreaming process, including a similarity solution for the flow inside and outside the droplet that leads to realistic surface tension gradients along the interface.

We desire a simplified model akin to the analytical model presented by Krechetnikov and Homsy that results in

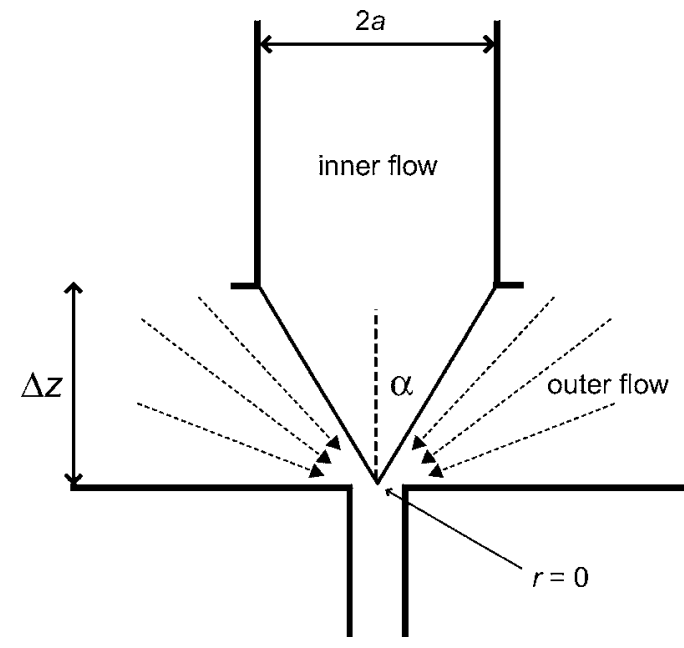

FIG. 12. Schematic diagram of a simplified model for the inner and outer flows in the microfluidic flow focusing device during thread formation and tipstreaming. The model assumes the tip remains stationary and thus the interface forms a cone with half-angle $\alpha$. The origin $(r=0)$ is taken at the tip of the cone, which also coincides with the entrance to the orifice.

scaling arguments for the dependence of the tipstreaming drop size and other measurable quantities on parameters such as flow rate ratio, capillary number, surfactant properties, and device geometry. While the details of such a model are beyond the scope of the present paper, we discuss here some experimental observations suggesting a route to a simplified model. Specifically, we performed a simple flow visualization experiment in which we seeded the inner aqueous stream with fluorescent particles and observed the motion of these particles inside the pointed droplet during thread formation. Figure 11 shows a time-lapse image of the seeded flow, resulting in images of the particle streamlines. In addition, the image is superimposed upon a brightfield image of the oil-water interface during the same time period. The brightfield image has been converted to black-and-white via a threshold operation. The image illustrates a number of key features of the thread formation and subsequent tipstreaming regime. During the period in which a thread is formed, the oil-water interface is stationary, forming a nearly perfect triangular shape with a fixed angle of $2 \alpha=49^{\circ}$, where $\alpha$ is the half-angle of the corner. In fact, this angle corresponds closely to the angle formed between the upstream corners at the end of the inner microchannel and the downstream entrance point of the orifice, as illustrated schematically in Fig. 12. The geometry of the flow focusing device suggests that the angle of such a corner is given by

$$
\alpha=\tan ^{-1}\left(\frac{a}{\Delta z}\right)=53.1^{\circ} \text {. }
$$

The measured cone angle is slightly smaller than that predicted from Eq. (8), most likely because the point of the cone is actually slightly inside the orifice rather than just at the entrance. A thin thread is drawn from the end of the stationary tip, as indicated by the white stream extending from the tip in the thresholded image. In addition to the shape of the oil-water interface, the traces of the fluorescent seed particles show that in the corner region, the inner liquid undergoes 
significant circulation. Two vortices are observed that are symmetric about the axis of the flow focusing device.

The flow visualization image of Fig. 11 suggests that an analytical solution for the thread formation and tipstreaming process may be accessible if one assumes that the Stokes equations are valid both inside and outside the oil-water interface, and that a two-dimensional solution is valid since dramatic changes in the flow-focusing geometry only occur in the plane. We seek solutions to the biharmonic equation in each liquid, ensuring that the outer flow corresponds to a flow similar to the driving flow in the actual device, and then we match boundary conditions to obtain the resulting surface tension gradient along the interface. A schematic diagram illustrating a simplified model for the process is shown in Fig. 12, in which the origin $r=0$ is defined at the entrance to the orifice. We postulate that planar flow into a slit is a reasonable driving flow in the outer liquid and should capture many of the essential features of the elongational flow. The inner flow resembles a Stokes corner flow. ${ }^{50}$ Planar flow into a slit is purely radial, ${ }^{51}$ leading to a solution for the velocity field of the form

$$
u_{r}^{\text {out }}(r, \theta) \sim \frac{Q_{C} \cos ^{2} \theta}{r} f(\alpha),
$$

where $f(\alpha)$ is a function of the corner angle and thus is also a function of the flow focusing geometry via Eq. (8). The form of the solution given above reflects the requirement that mass is conserved in the outer liquid. The radial velocity profile given in Eq. (9) requires that the shear stress at the oil-water interface have the form

$$
\left.\tau_{r \theta}\right|_{\theta=\alpha} \sim \frac{1}{r^{2}},
$$

which then leads to a surface tension profile along the interface with the form

$$
\gamma(r) \sim \gamma_{0}-\frac{Q_{C} g(\alpha)}{r},
$$

where $g(\alpha)$ is a positive function of the corner angle and $\gamma_{0}$ is the surface tension of a clean oil-water interface. This model is overly simplistic in many respects. For example, the radial velocity profile resulting from the slit flow assumption also requires that the inner fluid exhibit a purely radial velocity, eliminating the possibility for obtaining circulation flow that we know exists based on the experiment shown in Fig. 11. Furthermore, the internal circulation will complicate the transport of surfactants dissolved in the inner liquid to the interface. On the other hand, the surface tension profile along the interface exhibits the expected behavior in that the surface tension decreases as $r \rightarrow 0$, in other words, as we approach the tip of the interface.

Ultimately we wish to obtain predictions for the tipstreaming droplet size and other measurable quantities related to the thread formation and tipstreaming processes. In order to do that successfully, it will be necessary to connect the surface tension gradient as predicted in Eq. (11) with the formation of the thin thread. This raises questions as to whether the surface tension is bounded at dynamic interfaces as it is at static interfaces, ${ }^{46}$ and under which conditions the viscous stresses can overcome surface tension and draw out a thin thread. ${ }^{49}$ In addition, it will be necessary to account for the periodic nature of the process by incorporating the removal of surfactants from the interface via the thin thread and the time scales for the diffusion, adsorption, and desorption processes that enable surfactant to repopulate the interface in a finite time.

\section{CONCLUSIONS}

We report experimental observations of droplet formation in a microfluidic flow focusing device in which we systematically vary the capillary number, based upon the flow rate of the outer liquid stream, and the ratio of the outer liquid flow rate to the inner liquid flow rate. In addition, we systematically vary the bulk concentration of surfactant dissolved in the inner liquid phase, from zero up to concentrations on the order of the critical micelle concentration. Consistent with similar observations reported in the literature, we observe a progression of droplet breakup modes as the capillary number increases. At low capillary numbers, the dimensions of the flow focusing orifice control the pinchoff of droplets ("geometry-controlled"). As the capillary number increases, the inner liquid finger narrows due to viscous stresses from the outer liquid, and the resulting droplet sizes are somewhat smaller than the orifice width ("dripping"). At the highest capillary numbers, the inner liquid draws into a long jet that extended downstream of the orifice ("jetting") resulting in larger and less controlled droplet sizes. On the other hand, the transitions between these regimes are only weakly dependent on the flow rate ratio, suggesting that this parameter is not very important in controlling the droplet breakup mode. As we describe below, however, flow rate ratio is quite important in the dynamics of formation of fine threads when surfactants are present. All experiments reported are for a fixed viscosity ratio and a fixed choice of the dissolved surfactant.

In the presence of surfactants, a new droplet breakup mode called "thread formation" is observed and the behavior in this regime is strongly dependent upon the flow parameters and the surfactant bulk concentration. Thread formation occurs in a capillary number range of $0.4 \leq \mathrm{Ca} \leq 1.0$, coincident with the region in which tipstreaming occurs in experiments involving isolated droplets. ${ }^{17,20}$ The length of the thin threads increases as the outer liquid flows increasingly faster than the inner liquid, and exhibits a maximum at a capillary number of $\mathrm{Ca} \approx 0.5$. Measurements of the period for formation of primary droplets are consistent with these observations. At very large flow rate ratios, thread formation gives way to tipstreaming of micrometer-scale droplets. This observation is consistent with "classic" tipstreaming behavior in which the inner, droplet phase is stationary. On the other hand, we note that the bulk surfactant concentrations at which we observe thread formation are typically larger than the critical micelle concentration. This is in contrast to published experimental and theoretical studies that report much more moderate surface coverage conditions. We observe via a simple flow visualization experiment that the oil-water in- 
terface forms a stationary conical shape during thread formation and that within this corner, the inner fluid undergoes significant circulation. Based on these observations, we describe preliminary efforts at developing a simplified model for the tipstreaming process, which ultimately will need to include mass transfer mechanisms such as diffusion, adsorption, and desorption for transporting soluble surfactants from the bulk liquid to the interface, and possibly micellar dynamics.

The observations we present here are promising for the eventual production of micrometer- and submicrometer-scale droplets via microfluidic methods. Key advantages for using surfactant-mediated microfluidic methods include (a) reducing the parent droplet size in order to reduce the tipstreaming droplet size; (b) enabling continuous production of submicrometer droplets; (c) enabling production of droplets that are not limited by the minimum device feature size; and (d) enabling control of the depletion of surfactants from the interface by offering a continuous supply of surfactant molecules. Microfluidic flow focusing experiments appear to offer a unique ability to investigate tipstreaming mechanisms in detail. A detailed understanding of these mechanisms will enable control over the process, which will, in turn, offer the potential for application in areas such as nanoparticle synthesis, drug and gene delivery, and catalysis.

\section{ACKNOWLEDGMENTS}

Acknowledgment is made to the Donors of the American Chemical Society Petroleum Research Fund for partial support of this research. In addition, we acknowledge partial support from the Berkman Faculty Development Fund. We thank Kate Stebe, Howard Stone, Lynn Walker, Bud Homsy, and Steve Garoff for insightful discussions. We thank the Carnegie Mellon University MEMS Laboratory for use of facilities to fabricate devices, and we thank the Carnegie Mellon University Colloids, Polymers and Surfaces Laboratory for the use of surface characterization equipment.

${ }^{1}$ S. L. Anna, N. Bontoux, and H. A. Stone, "Formation of dispersions using 'flow focusing' in microchannels," Appl. Phys. Lett. 82, 364 (2003).

${ }^{2}$ T. Thorsen, R. W. Roberts, F. H. Arnold, and S. R. Quake, "Dynamic pattern formation in a vesicle-generating microfluidic device," Phys. Rev. Lett. 86, 4163 (2001).

${ }^{3}$ J. D. Tice, H. Song, A. D. Lyon, and R. F. Ismagilov, "Formation of droplets and mixing in multiphase microfluidics at low values of the Reynolds and the capillary numbers," Langmuir 19, 9127 (2003).

${ }^{4}$ P. Garstecki, I. Gitlin, W. DiLuzio, G. M. Whitesides, E. Kumacheva, and H. A. Stone, "Formation of monodisperse bubbles in a microfluidic flowfocusing device," Appl. Phys. Lett. 85, 2649 (2004).

${ }^{5}$ T. Cubaud, M. Tatineni, X. L. Zhong, and C. M. Ho, "Bubble dispenser in microfluidic devices," Phys. Rev. E 72, 037302 (2005).

${ }^{6}$ S. Q. Xu, Z. H. Nie, M. Seo, P. Lewis, E. Kumacheva, H. A. Stone, P. Garstecki, D. B. Weibel, I. Gitlin, and G. M. Whitesides, "Generation of monodisperse particles by using microfluidics: Control over size, shape, and composition," Angew. Chem., Int. Ed. 44, 724 (2005).

${ }^{7}$ D. Dendukuri, K. Tsoi, T. A. Hatton, and P. S. Doyle, "Controlled synthesis of nonspherical microparticles using microfluidics," Langmuir 21, 2113 (2005)

${ }^{8}$ T. Nisisako, T. Torii, and T. Higuchi, "Novel microreactors for functional polymer beads," Chem. Eng. J. 101, 23 (2004).
${ }^{9}$ A. S. Utada, E. Lorenceau, D. R. Link, P. D. Kaplan, H. A. Stone, and D. A. Weitz, "Monodisperse double emulsions generated from a microcapillary device," Science 308, 537 (2005).

${ }^{10}$ D. Dendukuri, D. C. Pregibon, J. Collins, T. A. Hatton, and P. S. Doyle, "Continuous flow lithography for high-throughput microparticle synthesis," Nat. Mater. 5, 365 (2006).

${ }^{11}$ A. B. Subramaniam, M. Abkarian, and H. A. Stone, "Controlled assembly of jammed colloidal shells on fluid droplets," Nat. Mater. 4, 553 (2005).

${ }^{12}$ F. J. Zendejas, U. Srinivasan, W. J. Holtz, J. D. Keasling, and R. T. Howe, "Microfluidic generation of tunable emulsions for templated monodisperse silica," International Conference on Solid State Sensors and Actuators and Microsystems, TRANSDUCERS '05 (2005), Vol. 2, p. 1473.

${ }^{13}$ P. Garstecki, H. A. Stone, and G. M. Whitesides, "Mechanism for flowrate controlled breakup in confined geometries: A route to monodisperse emulsions," Phys. Rev. Lett. 94, 164501 (2005).

${ }^{14}$ M. Y. He, J. S. Kuo, and D. T. Chiu, "Electro-generation of single femtoliter- and picoliter-volume aqueous droplets in microfluidic systems," Appl. Phys. Lett. 87, 031916 (2005).

${ }^{15}$ O. A. Basaran, "Small-scale free surface flows with breakup: Drop formation and emerging applications," AIChE J. 48, 1842 (2002).

${ }^{16}$ D. R. Link, S. L. Anna, D. A. Weitz, and H. A. Stone, "Geometrically mediated breakup of drops in microfluidic devices," Phys. Rev. Lett. 92, 054503 (2004).

${ }^{17}$ G. I. Taylor, "The formation of emulsions in definable fields of flow," Proc. R. Soc. London, Ser. A 146, 501 (1934).

${ }^{18} \mathrm{H}$. A. Stone, "Dynamics of drop deformation and breakup in viscous fluids," Annu. Rev. Fluid Mech. 26, 65 (1994)

${ }^{19}$ F. D. Rumscheidt and S. G. Mason, "Particle motions in sheared suspensions XII. Deformation and burst of fluid droplets in shear and hyperbolic flow," J. Colloid Sci. 16, 238 (1961).

${ }^{20}$ R. A. de Bruijn, "Tip-streaming of drops in simple shear flows," Chem. Eng. Sci. 48, 277 (1993).

${ }^{21}$ J. J. Janssen, A. Boon, and W. G. M. Agterof, "Influence of dynamic interfacial properties on droplet breakup in simple shear flow," AIChE J. 40, 1929 (1994).

${ }^{22}$ J. J. Janssen, A. Boon, and W. G. M. Agterof, "Influence of dynamic interfacial properties on droplet breakup in plane hyperbolic flow," $\mathrm{AIChE}$ J. 43, 1436 (1997).

${ }^{23}$ J. J. M. Janssen, A. Boon, and W. G. H. Agterof, "Droplet break-up in simple shear-flow in the presence of emulsifiers," Colloids Surf., A 91, 141 (1994).

${ }^{24}$ Y. T. Hu, D. J. Pine, and L. G. Leal, "Drop deformation, breakup, and coalescence with compatibilizer," Phys. Fluids 12, 484 (2000).

${ }^{25}$ C. D. Eggleton, T. M. Tsai, and K. J. Stebe, "Tip streaming from a drop in the presence of surfactants," Phys. Rev. Lett. 87, 048302 (2001).

${ }^{26} \mathrm{H}$. A. Stone and L. G. Leal, "The effects of surfactants on drop deformation and breakup," J. Fluid Mech. 220, 161 (1990).

${ }^{27}$ W. J. Milliken, H. A. Stone, and L. G. Leal, "The effect of surfactant on the transient motion of Newtonian drops," Phys. Fluids A 5, 69 (1993).

${ }^{28}$ I. B. Bazhlekov, P. D. Anderson, and H. E. H. Meijer, "Numerical investigation of the effect of insoluble surfactants on drop deformation and breakup in simple shear flow," J. Colloid Interface Sci. 298, 369 (2006).

${ }^{29}$ H. P. Grace, "Dispersion phenomena in high-viscosity immiscible fluid systems and application of static mixers as dispersion devices in such systems," Chem. Eng. Commun. 14, 225 (1982).

${ }^{30}$ M. R. Booty and M. Siegel, "Steady deformation and tip-streaming of a slender bubble with surfactant in an extensional flow," J. Fluid Mech. 544, 243 (2005).

${ }^{31}$ J. Eastoe and J. S. Dalton, "Dynamic surface tension and adsorption mechanisms of surfactants at the air-water interface," Adv. Colloid Interface Sci. 85, 103 (2000).

${ }^{32}$ A. M. Ganan-Calvo, "Perfectly monodisperse microbubbling by capillary flow focusing: An alternate physical description and universal scaling," Phys. Rev. E 69, 027301 (2004).

${ }^{33}$ D. C. Duffy, J. C. McDonald, O. J. A. Schueller, and G. M. Whitesides, "Rapid prototyping of microfluidic systems in poly(dimethylsiloxane)," Anal. Chem. 70, 4974 (1998).

${ }^{34}$ G. M. Whitesides and A. D. Stroock, "Flexible methods for microfluidics," Phys. Today 54 (6), 42 (2001).

${ }^{35}$ C. Donzel, M. Geissler, A. Bernard, H. Wolf, B. Michel, J. Hilborn, and E. Delamarche, "Hydrophilic poly(dimethylsiloxane) stamps for microcontact printing," Adv. Mater. (Weinheim, Ger.) 13, 1164 (2001).

${ }^{36}$ V. B. Fainerman, D. Mobius, and R. Miller, Surfactants: Chemistry, Interfacial Properties, Applications (Elsevier, Amsterdam, 2001). 
${ }^{37}$ F. Jin, R. Balasubramaniam, and K. J. Stebe, "Surfactant adsorption to spherical particles: The intrinsic length scale governing the shift from diffusion to kinetic-controlled mass transfer," J. Adhes. 80, 773 (2004).

${ }^{38}$ R. Pan, J. Green, and C. Maldarelli, "Theory and experiment on the measurement of kinetic rate constants for surfactant exchange at an air/water interface," J. Colloid Interface Sci. 205, 213 (1998).

${ }^{39}$ J. K. Ferri and K. J. Stebe, "Which surfactants reduce surface tension faster? A scaling argument for diffusion-controlled adsorption," Adv. Colloid Interface Sci. 85, 61 (2000).

${ }^{40}$ P. Mukherjee and K. J. Mysels, U.S. Department of Commerce, Washington, D.C., Report No. NSRDS-NBS 36, 1971.

${ }^{41}$ S. Y. Lin, R. Y. Tsay, L. W. Lin, and S. I. Chen, "Adsorption kinetics of $\mathrm{C}(12) \mathrm{E}(8)$ at the air-water interface: Adsorption onto a clean interface," Langmuir 12, 6530 (1996).

${ }^{42} \mathrm{~T}$. Matsumoto and H. Zenkoh, "Micelle structure in isotropic aqueous colloids of a poly(oxyethylene) amphiphile C12E8," Colloid Polym. Sci. 268, 536 (1990).

${ }^{43}$ Q. Y. Xu and M. Nakajima, "The generation of highly monodisperse droplets through the breakup of hydrodynamically focused microthread in a microfluidic device,” Appl. Phys. Lett. 85, 3726 (2004).
${ }^{44}$ L. Rayleigh, "On the capillary phenomena of jets," Proc. R. Soc. London 29, 71 (1879).

${ }^{45} \mathrm{~S}$. Tomotika, "On the instability of a cylindrical thread of a viscous liquid surrounded by another viscous fluid," Proc. R. Soc. London, Ser. A 150, $322(1935)$.

${ }^{46}$ F. Jin, N. R. Gupta, and K. J. Stebe, "The detachment of a viscous drop in a viscous solution in the presence of a soluble surfactant," Phys. Fluids 18, 022103 (2006).

${ }^{47}$ B. Ambravaneswaran and O. A. Basaran, "Effects of insoluble surfactants on the nonlinear deformation and breakup of stretching liquid bridges," Phys. Fluids 11, 997 (1999).

${ }^{48}$ J. M. Fernandez and G. M. Homsy, "Chemical reaction-driven tipstreaming phenomena in a pendant drop," Phys. Fluids 16, 2548 (2004).

${ }^{49}$ R. Krechetnikov and G. M. Homsy, "On physical mechanisms in chemical reaction-driven tip-streaming," Phys. Fluids 16, 2556 (2004).

${ }^{50}$ H. K. Moffatt, "Viscous and resistive eddies near a sharp corner," J. Fluid Mech. 18, 1 (1964).

${ }^{51}$ R. B. Bird, R. C. Armstrong, and O. Hassager, Dynamics of Polymeric Liquids. Volume 1: Fluid Mechanics, 2nd ed. (John Wiley \& Sons, New York, 1987). 